\title{
Tobacco use in Bollywood movies, tobacco promotional activities and their association with tobacco use among Indian adolescents
}

\author{
Monika Arora, ${ }^{1,2}$ Neha Mathur, ${ }^{1}$ Vinay K Gupta, ${ }^{1}$ Gaurang P Nazar, ${ }^{1}$ \\ K Srinath Reddy, ${ }^{1,2}$ James D Sargent ${ }^{3}$
}

${ }^{1}$ Health Related Information Dissemination Amongst Youth (HRIDAY), New Delhi, India ${ }^{2}$ Public Health Foundation of India (PHFI), New Delhi, India ${ }^{3}$ Community and Family Medicine and Pediatrics, Dartmouth Medical School, Hanover, New Hampshire, USA

Correspondence to Dr Monika Arora, HRIDAY, C-1/52, 3rd floor, Safdarjung Development Area, New Delhi 110016, India:

monika@hriday-shan.org

Received 7 February 2011 Accepted 8 June 2011

Published Online First

5 July 2011

\section{(2) UNLOCK:}

This paper is freely available online under the BMJ Journals unlocked scheme, see http:// tobaccocontrol.bmi.com/site/ about/unlocked.xhtml

\begin{abstract}
Background Smoking in Hollywood movies is a known risk factor for teen smoking in the USA and Europe, but little is known about the association between exposure to tobacco use in Bollywood movies and teen tobacco use in India.

Methods A cross-sectional sample of 3956 adolescents (eighth and ninth grades, ages 12-16 years) from 12 randomly selected New Delhi schools was surveyed in 2009, assessing tobacco use status, receptivity to tobacco promotions (based on owning or being willing to wear tobacco-branded merchandise) and exposure to tobacco use in movies. Quartiles of exposure to tobacco use in popular Bollywood movies released from 2006 to $2008(n=59)$ were determined by content coding them for tobacco use and querying the adolescents whether they had seen each one. Logistic regression was used to control for covariates including age, gender, parent education, school performance, sensation-seeking propensity, family and peer tobacco use, and authoritative parenting.
\end{abstract}

Results Altogether, the 59 movies contained 412 tobacco use occurrences. The prevalence of ever tobacco use among adolescents was 5.3\%. Compared with low-exposure adolescents (quartile 1), the adjusted odds of ever tobacco use among high-exposure adolescents (quartile 4) was 2.3 (95\% Cl 1.3 to 3.9 ). Being receptive to tobacco promotions was also associated with higher adjusted odds of ever tobacco use, $2.0(95 \% \mathrm{Cl} 1.4$ to 3.0$)$.

Conclusion Watching tobacco use in Bollywood movies and receptivity to tobacco promotional activities were both independently associated with ever tobacco use among adolescents in India, with ORs being similar to the studies of adolescents elsewhere.

\section{INTRODUCTION}

Globally, tobacco use continues to be the leading cause of preventable death, ${ }^{1}$ estimated to kill more than five million people annually worldwide. The epidemic of tobacco use is shifting from developed to developing countries. ${ }^{2}$ It is estimated that by 2030, 10 million people per year will die from tobacco use, with $70 \%$ of those deaths occurring in developing countries. ${ }^{3}$ India accounts for one-sixth of tobacco illnesses worldwide and will face an exponential increase in tobacco-related mortality from $1.4 \%$ of all deaths in 1990 to $13.3 \%$ in $2020 .{ }^{4}$

Smoking in Hollywood (metonym for the American movie industry) movies is a known risk factor for teen smoking in the USA ${ }^{5}$ and Europe. ${ }^{6}$
India, the world's largest producer of movies, produces more than 1000 movies a year in several different languages when compared with around 500 in the USA. Bollywood represents the Indian Hindi movie industry, and in 2005, around $24 \%$ of the Indian movies produced were in Hindi language. ${ }^{7}$ Indian movies are watched by over 188 million Indian viewers every year and are distributed to several nations including the Middle-East, South East Asia, USA, UK and Europe. ${ }^{8}$ The worldwide viewership for Bollywood movies has been estimated to be about 3 million when compared with 2.6 million for Hollywood movies. ${ }^{9}$ Bollywood movie stars in India lead public lives, have large fan followings and therefore could influence the behaviour and attitudes of adolescents there. $^{8}$ Yet there are no studies that test the hypothesis that there is a relation between watching tobacco use in Bollywood movies and tobacco use among Indian adolescents. Moreover, primary research has suggested that exposure to tobacco marketing is associated with increased tobacco use among Indian adolescents, ${ }^{10}{ }^{11}$ but there are no studies that assess ownership of tobacco-branded merchandise. Both types of promotions (tobacco use by celebrities in movies and the distribution of tobacco-branded merchandise) should have been eliminated by the enactment of the Indian tobacco control law, 'The Cigarettes and Other Tobacco Products (Prohibition of Advertisement and Regulation of Trade and Commerce, Production, Supply and Distribution) Act, 2003' (COTPA, 2003). To determine whether adolescents were exposed to these risk factors and whether such exposure was associated with youth tobacco use, we conducted a cross-sectional study among Indian school-going adolescents.

\section{METHODS}

\section{Study design and setting}

This school-based cross-sectional study was conducted in 2009 in 12 randomly selected senior secondary schools in New Delhi. From the list of 3524 schools under the Directorate of Education, Government of National Capital Territory of Delhi, 100 schools with an active ongoing health education programme on tobacco prevention were excluded. Out of 3424 schools in the sampling frame, 36 were randomly selected including 18 private and 18 government schools. Of these 36 schools, 12 consented to participate in the study, including 6 private and 6 government schools. Government schools were considered to be catering 
to low socio-economic status (SES) groups and private schools to high SES groups as the cost to send students to private schools is much higher than in the government schools, which either have a nominal fee or offer free education. The school type was used as a proxy measure for SES in this study in accordance with earlier similar studies in India. ${ }^{12}$

\section{Participants}

A total sample of 4943 adolescents from eighth and ninth grades (age 12-16 years) were eligible for the survey. An active informed consent procedure was followed in which informed consent from schools and parents along with students' assents were obtained after assuring confidentiality. Out of 4943 eligible adolescents, 3956 were surveyed with a survey response rate of $80 \%$. Of those missing from the survey, $11.1 \%$ were due to parents refusing participation of their child, $4.4 \%$ dropped out (before/during survey administration), $4.2 \%$ were absent on the day of survey and $0.3 \%$ adolescents refused to participate. Ethics approval for research on human subjects for this study was obtained from the Independent Ethics Committee (IEC), Mumbai.

\section{Data sources and variables Survey instrument}

The survey instrument was culturally adapted for India from a questionnaire used in previous studies ${ }^{13}$ and translated into Hindi language for administration in low SES schools, in part based on a previously validated instrument. ${ }^{14}$ The paper-andpencil-based, self-administered questionnaire was implemented in the classrooms by teams using a standardised protocol. Confidentiality was maintained by detaching the student assent form (containing the name of the student) from the survey questionnaire before the students started filling in their responses to the survey questions. Teachers stayed in the class to maintain order, but they were not allowed to see the student responses.

\section{Exposure assessment}

Exposure to tobacco use in movies was assessed by asking the adolescents whether they had seen any movie from the fixed list of 59 Bollywood movies. Previously, adolescents have reported on movies they have seen with high accuracy. ${ }^{15}$ Box office reports were used to assess the success levels of the movies. The selection criteria included, top grossing 15 movies per year for 3 years (2006-2008) and four to five movies per year that were known to have extensive depiction of tobacco use. Occurrences of tobacco use in the movies were counted as an assessment of the extent to which tobacco was portrayed. A tobacco occurrence was defined as the appearance of a tobacco product being used by a character on screen (cigarettes, beedi, smokeless tobacco, cigar, pipe, smoky background and an appearance of a tobacco product with ownership by a particular character). In a given scene, the number of occurrences was capped at 10 and dictated by the number of characters within the scene who used or handled a tobacco product. Inter-rater reliability was addressed by comparing tobacco occurrences for six movies by coders in the USA and in India as per the coding protocol. The protocol was revised in a joint meeting of the two coding teams to resolve coding discrepancies. Out of the 59 movies on the list, $60 \%$ depicted the use of tobacco in one form or the other, with a mean of seven tobacco use occurrences in a movie. A few movies (eg, Traffic Signal, Fashion and Black Friday) had very high number of tobacco use occurrences. The primary exposure was total number of tobacco use occurrences the adolescents had seen from the list of 59 movies, parsed into population quartiles for the analysis.

\section{Receptivity to tobacco promotions}

Although the activity has been illegal in India since 2004, tobacco companies continue to distribute tobacco-branded merchandise (including T-shirts, caps, posters, belts, helmets and water bottles) through surrogate means, sold in retail shops and distributed through various other channels. For example, smokeless tobacco products are advertised through printing their pack shots and brand names on kites that children fly throughout India on Independence Day. Receptivity to tobacco promotions was assessed through the following two questions: 'Do you have an item (like a T-shirt, cap, poster, belt or water bottle) that bears a tobacco brand name or logo on it?' and 'Would you ever wear or use an item (like a T-shirt, cap, poster, belt or water bottle) that bears a tobacco brand name or logo on it?' Response options for both were 'Yes' (coded 1) and 'No' (coded 0). As with previous research, ${ }^{16}$ an affirmative response to either question was indicative of receptivity to the promotional programme.

\section{Outcome measure}

'Ever tobacco use' was assessed through the following question: 'How old were you when you first smoked or chewed tobacco in any form?' The response categories were 'I have never used tobacco' or a specific age ranging from 7 years or less to 15 years or more. Affirmative responses were collapsed to create a dichotomous variable.

\section{Covariates}

Based on previous studies, we controlled for socio-demographics (age, gender, self-reported school performance, sensation seeking ('I like to do scary things', 'I like to do dangerous things', 'I often think there is nothing to do', 'I like to listen to loud music', $\alpha=0.64),{ }^{17}$ family and peer tobacco use, authoritative parenting (parental responsiveness and demandingness) ('S/he is always telling me what to do, makes me feel better when I'm upset, listens to what I have to say, wants to hear about my problems' and 'S/he has rules that I must follow, makes sure I tell her/him where I am going, makes sure I go to bed on time, checks to see that I do my homework', $\alpha=0.82)$ ). ${ }^{18}$ The value of $\alpha$ (Cronbach's $\alpha)$ for sensation seeking and authoritative parenting shows that both the summative scales were internally consistent.

\section{Statistical methods}

Two-way associations between ever tobacco use or exposure to movie tobacco use and each of the other predictor variables were explored using $\chi^{2}$ test or analysis of variance. Multiple logistic regression analysis was used to determine the crude and adjusted ORs and 95\% CIs for association between ever tobacco use and exposure to tobacco use in movies. Indexed variables (sensation seeking and the authoritative parenting measures) were dichotomised using a median split. All tests were considered significant at the 0.05 level. Only complete cases were considered when the covariates were included in the models. All the analyses were done using statistical software SAS V.9.1.

\section{Non-response to survey items}

Non-response to one or more movie queries was an issue in this questionnaire with 1351 adolescents failing to respond to one or more movie questions. As non-response tended to result in lower scores for exposure to tobacco use in movies, a sensitivity analysis was conducted to determine how this may have 
affected estimates of the relation between tobacco use and exposure to tobacco use in movies. Two models were used for this purpose. In the first model, the students who did not respond to more than 20 movies $(n=198)$ were removed from the data. In the second model, any student who failed to respond to one or more movie items was removed $(n=1351)$. We examined how these alterations affected the crude dose-response between exposure and tobacco use, as well as the adjusted ORs in the fully adjusted model.

\section{RESULTS}

\section{Characteristics of the sample}

Table 1 shows the demographic profile of the respondents including age, gender, grade, SES, academic performance, tobacco use by family members and friends, mean values of exposure to tobacco occurrences, sensation-seeking behaviour and authoritative parenting. The overall prevalence of ever tobacco use among school-going adolescents (those who have consumed tobacco at least once in their lifetime) was found to be $5.3 \%$. This prevalence was significantly higher among boys $(6.2 \%)$ than girls $(4.3 \%)(\mathrm{p}<0.05)$, among lower SES group $(8.2 \%)$ than higher SES group $(3.1 \%)(p<0.001)$, and in older age group, as shown in table 1.

\section{Exposure to tobacco use in Bollywood movies and receptivity to tobacco promotions}

Adolescents had seen a mean of 31 out of 59 movies. Mean exposure of tobacco use occurrences was 162 , which was slightly higher than the median (144) (range 0-412 and IOR 86-228). With respect to tobacco promotions, $11.8 \%$ of adolescents were receptive, of which 283 (7.3\% of all students) responded that they owned a personal item that had a tobacco brand name or logo on it. Exposure to tobacco use in movies was significantly associated with the demographic profile of the adolescents and other risk factors such as social influences and characteristics of adolescent and parenting. Table 2 shows that boys had higher exposure than girls (177.2 (SE 2.1) vs 143.2 (SE 2.3); $\mathrm{p}<0.001$ ). Exposure was also higher for adolescents belonging to higher SES than lower SES (171.9 (SE 2.3) vs 148.5 (SE 2.1); $\mathrm{p}<0.001$ ), and among ninth graders compared with eighth graders (166.3 (SE 2.3) vs 157.3 (SE 2.2); $\mathrm{p}<0.01$ ). Those who were receptive to tobacco promotions or who had tobacco users as friends were also significantly more exposed to movie smoking. Finally, exposure was also significantly higher for those with higher level of sensation seeking and, surprisingly, for those with more authoritative parents.

\section{Exposure to tobacco use in Bollywood movies, receptivity to tobacco promotions and ever tobacco use}

Table 3 represents the crude and adjusted ORs to show the independent relationship between exposure to tobacco use in movies, receptivity to tobacco promotions and ever tobacco use among adolescents. In the model that adjusted only for receptivity to tobacco promotions, adolescents belonging to fourth quartile of exposure to tobacco use were 1.9 times more likely (95\% CI 1.3 to 2.9 ) to use tobacco than those belonging to first quartile. When demographic profiles were entered, the OR increased to 2.3 (95\% CI 1.5 to 3.6). Adjustment of other factors like social influences, characteristics of adolescent and parenting did not affect the results much (fully adjusted OR for quartile 4 vs quartile $1=2.3$ (95\% CI 1.3 to 3.9$)$ ). As a test for dose-response, we entered quartile as a continuous variable (with quartile 1 set at zero) and confirmed evidence of a dose-response, with an adjusted OR of 1.3 (95\% CI 1.1 to 1.5$)$
Table 1 Association between ever tobacco use and other variables among Indian adolescents $(\mathrm{n}=3956)$

\begin{tabular}{|c|c|c|c|}
\hline & n (\%) & $\begin{array}{l}\text { Ever tobacco } \\
\text { use }(\%)\end{array}$ & p Value \\
\hline Total & $3956(100.0)$ & $204(5.3)$ & \\
\hline \multicolumn{4}{|c|}{ Exposure to tobacco use in movies } \\
\hline First quartile $(0-86)$ & $1010(25.5)$ & $42(4.3)$ & $<0.001$ \\
\hline Second quartile (87-144) & $972(24.6)$ & $32(3.4)$ & \\
\hline Third quartile (145-228) & $986(24.9)$ & $53(5.5)$ & \\
\hline Fourth quartile $(>228)$ & $988(25.0)$ & $77(8.0)$ & \\
\hline \multicolumn{4}{|c|}{ Receptive to tobacco promotions } \\
\hline No & $3441(87.0)$ & $143(4.3)$ & $<0.001$ \\
\hline Yes & $466(11.8)$ & $53(11.8)$ & \\
\hline Missing responses & $49(1.2)$ & & \\
\hline \multicolumn{4}{|l|}{ Demographic profile } \\
\hline \multicolumn{4}{|l|}{ Age (years) } \\
\hline$\leq 13$ & $1239(31.3)$ & $50(4.1)$ & $<0.001$ \\
\hline 14 & $1396(35.3)$ & $54(4.0)$ & \\
\hline 15 & $718(18.2)$ & $42(6.1)$ & \\
\hline 16 and above & $420(10.6)$ & $38(9.6)$ & \\
\hline Missing responses & $183(4.6)$ & & \\
\hline \multicolumn{4}{|l|}{ Gender } \\
\hline Boy & $2141(54.1)$ & $128(6.2)$ & 0.011 \\
\hline Girl & $1815(45.9)$ & $76(4.3)$ & \\
\hline \multicolumn{4}{|l|}{ Socio-economic status } \\
\hline High (private schools) & $2208(55.8)$ & $68(3.1)$ & $<0.001$ \\
\hline Low (government) & $1748(44.2)$ & $136(8.2)$ & \\
\hline \multicolumn{4}{|l|}{ Class } \\
\hline Eighth & $1886(47.7)$ & $118(5.9)$ & 0.087 \\
\hline Ninth & $2070(52.3)$ & $86(4.8)$ & \\
\hline \multicolumn{4}{|l|}{ Social influences } \\
\hline \multicolumn{4}{|l|}{ Family members use tobacco } \\
\hline No & $2635(66.6)$ & $98(3.8)$ & $<0.001$ \\
\hline Yes & $1321(33.4)$ & $106(8.3)$ & \\
\hline \multicolumn{4}{|l|}{ Friends using tobacco } \\
\hline No & $3277(82.8)$ & $93(2.9)$ & $<0.001$ \\
\hline Yes & $533(13.5)$ & $99(19.2)$ & \\
\hline Missing responses & $146(3.7)$ & & \\
\hline \multicolumn{4}{|c|}{ Characteristics of child and parenting } \\
\hline \multicolumn{4}{|l|}{ Academic performance } \\
\hline Excellent & $902(22.8)$ & $24(2.7)$ & $<0.001$ \\
\hline Good & $1410(35.7)$ & $65(4.7)$ & \\
\hline Average & $1012(25.6)$ & $62(6.4)$ & \\
\hline Below average & $497(12.6)$ & $40(8.5)$ & \\
\hline Missing responses & $135(3.4)$ & & \\
\hline \multicolumn{4}{|l|}{ Sensation seeking } \\
\hline Lower score & $2104(53.2)$ & $107(5.3)$ & 0.975 \\
\hline Higher score & $1852(46.8)$ & $97(5.3)$ & \\
\hline \multicolumn{4}{|c|}{ Parental responsiveness and demandingness } \\
\hline Lower score & $2004(50.7)$ & $110(5.7)$ & 0.293 \\
\hline Higher score & $1952(49.3)$ & $94(4.9)$ & \\
\hline
\end{tabular}

(data not shown). Adolescents receptive to tobacco promotions were three times more likely to use tobacco (95\% CI 2.1 to 4.2 ) in the model that adjusted only for movie tobacco exposure. The fully adjusted OR of using tobacco given receptivity to tobacco marketing was 2.0 (95\% CI 1.4 to 3.0 ).

\section{Sensitivity analysis}

The Lowess curve (figure 1) illustrates the dose-response relationship between exposure to movie tobacco use and prevalence of ever tobacco use, showing that when the full sample was assessed, there was a decrease in the prevalence of ever tobacco use up to an exposure to 100 occurrences, followed by an increase in prevalence at higher levels of exposure. The $95 \% \mathrm{CI}$ 
Table 2 Association between mean exposure to tobacco use in movies and other variables for Indian adolescents $(n=3956)$

\begin{tabular}{|c|c|c|}
\hline & Mean exposure (SE) & p Value \\
\hline \multicolumn{3}{|c|}{ Socio-demographic profile } \\
\hline \multicolumn{3}{|l|}{ Age (years) } \\
\hline$\leq 13$ & $153.8(2.8)$ & \multirow[t]{4}{*}{0.001} \\
\hline 14 & $163.1(2.6)$ & \\
\hline 15 & $171.4(3.6)$ & \\
\hline 16 and above & $166.1(4.8)$ & \\
\hline \multicolumn{3}{|l|}{ Gender } \\
\hline Boy & $177.2(2.1)$ & \multirow[t]{2}{*}{$<0.001$} \\
\hline Girl & $143.2(2.3)$ & \\
\hline \multicolumn{3}{|l|}{ School type } \\
\hline High SES & $171.9(2.3)$ & \multirow[t]{2}{*}{$<0.001$} \\
\hline Low SES & $148.5(2.1)$ & \\
\hline \multicolumn{3}{|l|}{ Class } \\
\hline Eighth & $157.3(2.2)$ & \multirow[t]{2}{*}{0.004} \\
\hline Ninth & $166.3(2.3)$ & \\
\hline \multicolumn{3}{|l|}{ Social influences } \\
\hline \multicolumn{3}{|c|}{ Receptive to tobacco promotions } \\
\hline No & $159.1(1.7)$ & \multirow[t]{2}{*}{$<0.001$} \\
\hline Yes & $184.6(4.5)$ & \\
\hline \multicolumn{3}{|c|}{ Family members using tobacco } \\
\hline No & $164.2(1.9)$ & \multirow[t]{2}{*}{0.017} \\
\hline Yes & $156.3(2.7)$ & \\
\hline \multicolumn{3}{|c|}{ Friends using tobacco } \\
\hline No & $156.6(1.7)$ & \multirow[t]{2}{*}{$<0.001$} \\
\hline Yes & $201.4(4.2)$ & \\
\hline \multicolumn{3}{|c|}{ Characteristics of child and parenting } \\
\hline \multicolumn{3}{|c|}{ Academic performance } \\
\hline Excellent & $157.7(3.3)$ & \multirow[t]{4}{*}{$<0.001$} \\
\hline Good & $170.6(2.6)$ & \\
\hline Average & $161.4(3.1)$ & \\
\hline Below average & $151.4(4.4)$ & \\
\hline \multicolumn{3}{|c|}{ Sensation seeking } \\
\hline Lower score & $143.5(2.1)$ & \multirow[t]{2}{*}{$<0.001$} \\
\hline Higher score & $184.0(2.2)$ & \\
\hline \multicolumn{3}{|c|}{ Parental responsiveness and demandingness } \\
\hline Lower score & $158.3(2.2)$ & \multirow[t]{2}{*}{0.031} \\
\hline Higher score & $165.0(2.2)$ & \\
\hline
\end{tabular}

SES, socio-economic status.

for the predicted values on Lowess curve followed similar curves (not shown in the figure). This may have been due to systematically underestimating exposure in adolescents who did not respond to all movie items, because the curve became more linear as the non-responders were removed from the sample. Table 4 shows results of the final model from table 3 and two other models to compare how unmeasured responses may have affected the association between tobacco use and exposure to tobacco use in movies.

\section{DISCUSSION}

Research from Western countries has provided strong evidence for an association between viewing smoking in movies and smoking initiation among adolescents there. ${ }^{19} 20$ The present study confirms a similar relation for Bollywood movies and tobacco use behaviour of school-going Indian adolescents. Even after adjusting for demographic characteristics, social influences and child as well as parenting characteristics, the odds of ever tobacco use was more than double for those with high exposure as compared with low exposure, findings that are very similar to an earlier study undertaken in USA, ${ }^{21}$ and elsewhere, but assessing exposure mainly in Hollywood movies.

Adolescents in this study had seen a mean of 162 tobacco use occurrences from the 59 movies. These findings suggest that scenes depicting tobacco use are shown in Bollywood movies even after the enactment of COTPA in 2004, Section 5, which prohibits direct and indirect forms of tobacco advertising. Amendment of rules related to Section 5 of COTPA in 2005 completely prohibited promotion and advertising of tobacco products through movies under the direction of Ministry of Health and Family Welfare (MoHFW). ${ }^{22}$ However, this move of the MoHFW was undermined by the legal action that followed, when movies-related subrules of COTPA Section 5 were challenged in the High Court by a Bollywood film producer and struck down in 2009 because of free speech concerns. In response to this decision, the Government of India has filed petition for special leave to appeal with the Supreme Court of India, to uphold strong regulatory measures related to depiction of tobacco use in movies in the interest of public health, a decision for which is pending. The results of this study provide support for the special leave to appeal.

Apart from this legal battle, the blanket ban on depiction of all tobacco products in movies imposed by the 2005 amendment of rules under Section 5 of COTPA was also opposed by the Ministry of Information and Broadcasting (MoIB), which recommended giving adult (A) certification to those movies for which there was creative justification for depicting tobacco use. $^{22}$ A subsequent notification in 2006 permitted display or use of tobacco products in films, provided strong editorial

Table 3 Association of ever tobacco use with exposure to tobacco use in movies and receptivity to tobacco promotions among Indian adolescents $(n=3956)$

\begin{tabular}{|c|c|c|c|c|}
\hline \multirow[b]{3}{*}{ Exposure variable } & \multicolumn{4}{|l|}{ OR $(95 \%$ Cl) } \\
\hline & \multirow[b]{2}{*}{ Crude } & \multicolumn{3}{|l|}{ Adjusted } \\
\hline & & Model I* & Model II† & Model III† \\
\hline \multicolumn{5}{|l|}{ Movie tobacco use } \\
\hline First quartile & Reference & Reference & Reference & Reference \\
\hline Second quartile & $0.8(0.5-1.3)$ & $1.0(0.6-1.7)$ & $1.0(0.6-1.8)$ & $1.1(0.6-2.0)$ \\
\hline Third quartile & $1.3(0.8-2.0)$ & $1.5(1.0-2.4)$ & $1.5(0.9-2.5)$ & $1.6(0.9-2.7)$ \\
\hline Fourth quartile & $1.9(1.3-2.9)$ & $2.3(1.5-3.6)$ & $2.1(1.3-3.5)$ & $2.3(1.3-3.9)$ \\
\hline \multicolumn{5}{|c|}{ Receptive to tobacco promotions } \\
\hline No & Reference & Reference & Reference & Reference \\
\hline Yes & $3.0(2.1-4.2)$ & $2.8(1.9-3.9)$ & $2.0(1.4-3.0)$ & $2.0(1.4-3.0)$ \\
\hline
\end{tabular}

*Adjusted for age, gender, school type and class.

†Adjusted for demographic profiles and receptive to tobacco advertisements, family members using tobacco and friends using tobacco.

$\ddagger$ Adjusted for demographic profiles, social influences, academic performance, sensation seeking and authoritative parenting. 


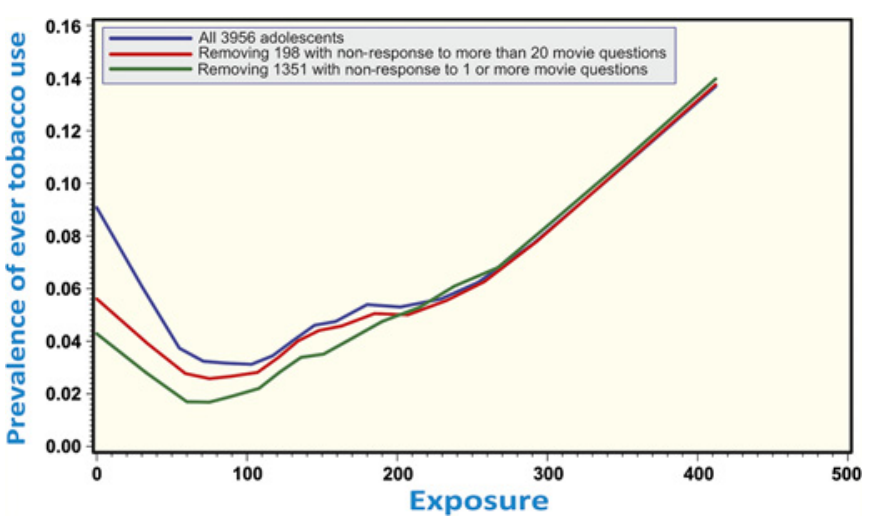

Figure 1 Lowess curve showing relationship between prevalence of ever tobacco use and exposure to tobacco use in Bollywood movies.

justification is given, and such films and television programmes will mandatorily be given ' $A$ ' certification. ${ }^{23}$ This notification was in accordance with the WHO recommendations on this issue, which allow such depiction in case of portrayal of a historical figure who used tobacco or when some negative message about tobacco use is to be conveyed. ${ }^{22}$ However, the 2006 rules have still not been implemented. Currently, the MoIB and MoHFW are in consultation to announce mutually agreeable rules for Section 5 of COTPA.

Beyond movie tobacco depictions, adolescents who owned or were willing to wear tobacco-branded merchandise (of whom a majority stated that they owned such a personal item) were two times more likely to have tried tobacco. This OR is almost identical to an earlier study undertaken with school-going Indian adolescents ${ }^{10}$ and multiple studies conducted in other countries. $^{24-26}$ India ratified the WHO Framework Convention on Tobacco Control (FCTC) on 5 February 2004. Section 5 of COTPA complies with the provisions prescribed under Article 13 of FCTC. Despite a comprehensive regulation, restricting tobacco promotions, $7.3 \%$ of all the students reported owning a tobacco promotional item in our study, which suggests that tobacco companies are violating the prescribed regulations for tobacco promotions under Section 5 of COTPA. Overall, this suggests that enforcement of Section 5 of COTPA needs to be strengthened.

The prevalence of ever tobacco use in our study for boys and girls was $6.2 \%$ and $4.3 \%$ respectively. These findings are similar to prevalence rates reported in an earlier study undertaken with North Indian adolescents. ${ }^{27}$ The results of this study are in accordance with previous studies in India, which suggest that ever tobacco use is higher among adolescents belonging to low SES group. ${ }^{12}$ Social influences such as having friends and family

Table 4 Sensitivity analysis showing how missing data for exposure to tobacco use in movies was handled for assessment of its relation with ever tobacco use

\begin{tabular}{llll}
\hline $\begin{array}{l}\text { Exposure to movie } \\
\text { tobacco use }\end{array}$ & \multicolumn{4}{l}{ Adjusted* OR $\mathbf{~ ( 9 5 \% ~ C l ) ~}$} \\
\cline { 2 - 4 } & Model I† & Model II & Model III§ \\
\hline First quartile & Reference & Reference & Reference \\
Second quartile & $1.1(0.6-2.0)$ & $1.3(0.7-2.6)$ & $1.7(0.7-4.0)$ \\
Third quartile & $1.6(0.9-2.7)$ & $2.0(1.1-3.7)$ & $1.8(0.8-4.1)$ \\
Fourth quartile & $2.3(1.3-3.9)$ & $2.9(1.6-5.3)$ & $3.6(1.7-7.6)$
\end{tabular}

*Adjusted for demographic profiles, social influences, academic performance, sensation seeking and authoritative parenting.

tFinal model from table 3 , no adjustment for missing data on movie tobacco use ( $n=3956)$. fDropping 198 students who failed to answer more than 20 movie questions $(n=3758)$.

§Dropping 1351 students who failed to answer one or more movie questions $(n=2605)$ members who use tobacco were significantly associated with ever tobacco use as shown in earlier literature. ${ }^{28}$ Prevalence of ever tobacco use was significantly higher among those adolescents who were highly receptive to tobacco promotions as reported in an earlier study undertaken with school-going adolescents. ${ }^{10}$ Results also suggest that boys are much more exposed than girls. Such findings, using content analysis of movies, have been reported for the first time in Indian context.

This study has some limitations. First, its cross-sectional design limits our ability to draw causal inferences; further longitudinal studies are required to assess whether exposure to tobacco use in Bollywood movies predicts onset of tobacco use behaviour. Second, the study's generalisability is limited to urban school-going adolescents, as non-school-going adolescents and adolescents from rural areas were not included. However, similar results have been demonstrated in earlier studies undertaken with white adolescents, ${ }^{6}$ even in rural settings, ${ }^{28}$ which suggests that the effect of exposure to tobacco use in movies on tobacco use behaviour of adolescents is not very different across cultures and settings. Third, in contrast to other studies, the prevalence of tobacco use decreased up to an exposure to 100 movie tobacco occurrences and increased thereafter. The sensitivity analysis raises possibility that survey item non-response could partially explain this phenomenon, and further study is required to determine whether the observed non-linear dose-response to tobacco use in movies is a real phenomenon in this population.

In summary, this study provides evidence that the association between seeing movie tobacco use and youth tobacco use is not restricted to Hollywood movies or Western countries. The findings support strengthening legal provisions in India to restrict depictions of tobacco use in Bollywood movies. The study also suggests that tobacco-branded merchandise still reaches adolescents some 6 years after enforcement of legislation that restricted tobacco promotion in all forms and supports efforts to bolster enforcement of COTPA in India.

\section{What this paper adds}

- Studies in developed countries have established that exposure to smoking in Hollywood movies leads to increased risk of smoking among adolescents. Although preliminary research in India suggests that tobacco marketing is associated with increased tobacco use, the impact of marketing strategies such as promotion through Bollywood movies and that of distribution of tobacco-branded merchandise on tobacco use among Indian adolescents has not been explored earlier.

- Adjusted odds of ever tobacco use among students who were highly exposed to tobacco use occurrences in Bollywood movies were more than twice when compared with those with low exposure. Similarly, students who owned or were willing to wear tobacco-branded merchandise had greater chances of being an ever tobacco user. These results suggest that the association between exposure to tobacco use occurrences in movies and tobacco use among adolescents is not restricted to Hollywood but also applies to Bollywood movies. Also, $7.3 \%$ of all adolescents in our study reported owning a tobacco promotional item, which suggests that tobacco companies are violating Section 5 of the Indian tobacco control law and calls for stricter implementation thereof. 
Acknowledgements We are thankful to the 12 schools that participated in this study. This research would not have been possible without the participation of adolescents and cooperation of teachers in the selected schools in New Delhi. The authors would also like to acknowledge the legal guidance provided by lawyers from our organisation, Ms Deepti Singh and Mr Amit Yadav, for this manuscript.

Funding This research was funded by a grant from Fogarty International Center, National Institutes of Health, Bethesda, Maryland, USA (award number 3R01TW007933-02S1; Monika Arora, principal investigator). Other funders: NIH.

\section{Competing interests None.}

Ethics approval This study was conducted with the approval of the Independent Ethics Committee, Mumbai (http://www.iecindia.org/default.htm; IEC/08/28).

Contributors MA is the principal investigator of the cross-sectional study entitled 'Association between tobacco use in Bollywood films and tobacco use among Indian adolescents'. She worked on all aspects of the study including concept and design, data collection protocols, data management and data analysis. She wrote portions of this manuscript, revised it critically for intellectual content and finalised the manuscript contents. NM is a research associate and was responsible for the management of the scientific components of this study. She was involved in data management and data analysis. She wrote sections in this research article pertaining to the introduction, methods and results. She was also involved in the formatting of this manuscript. VKG is the biostatistician and was involved in data analyses. He contributed to writing the methods and results section of the study. GPN is the project officer who was involved in the acquisition and interpretation of the data. He wrote sections in this manuscript pertaining to the discussion, contributed to revising it critically for intellectual content and formatting of the manuscript. KSR is the co-investigator (India) of this study and is responsible for the scientific integrity of the study as well as the study concept and design. He was involved in editing and finalising the manuscript content. JDS is the co-investigator (USA) of this study and is responsible for the scientific integrity of the study. He provided guidance to the Indian investigators in content coding of movies for tobacco use through the specialised coding procedure developed at Dartmouth Medical School (USA). He also contributed to the data analysis and results sections of the manuscript.

Provenance and peer review Not commissioned; externally peer reviewed.

\section{REFERENCES}

1. WHO. The Millennium Development Goals and Tobacco Control. Geneva: World Health Organization, 2005

2. Ezzati M, Lopez AD. Estimates of global mortality attributable to smoking in 2000 . Lancet 2003:362:847-52.

3. Stewart BW, Kleihues P, eds. World Cancer Report. Lyon: IARC Press, 2003

4. Reddy KS, Gupta PC. Report on Tobacco Control in India. New Delhi: Ministry of Health and Family Welfare, Government of India, 2004.

5. National Cancer Institute. The Role of the Media in Promoting and Reducing Tobacco Use. Tobacco Control Monograph No. 19. Bethesda, MD: U.S. Department of Health and Human Services, National Institutes of Health, National Cancer Institute, 2008

6. Hanewinkel R, Sargent JD. Exposure to smoking in internationally distributed American movies and youth smoking in Germany: a cross-cultural cohort study. Pediatrics 2008:121:e108-17.
7. UNESCO Institute for Statistics. Information Sheet No. 1. Analysis of the UIS International Survey on Feature Film Statistics. 2010. http://uissv13.uis.umontreal.ca/ culture/Documents/Infosheet No1 cinema EN.pdf (accessed 27 Jun 2011).

8. World Health Organisation. 'Bollywood': Victim or Ally? A WHO study on the Portrayal of Tobacco in Indian Cinema. Geneva: World Health Organization, 2003.

9. Worldpress. Bollywood vs. Hollywood. The Complete Breakdown. 2007. http:// www.mutiny.wordpress.com/2007/02/01/bollywood-vs-hollywood-the-completebreakdown (accessed 18 May 2011).

10. Arora M, Reddy KS, Stigler MH, et al. Associations between tobacco marketing and use among urban youth in India. Am J Health Behav 2008;32:283-94.

11. Viswanath K, Ackerson LK, Sorensen G, et al. Movies and TV influence tobacco use in India: findings from a national survey. PLoS One 2010:5:e11365.

12. Mathur C, Stigler MH, Perry CL, et al. Differences in prevalence of tobacco use among Indian Urban youth: the role of socioeconomic status. Nicotine Tob Res 2008:10:109-16

13. Sargent JD, Beach ML, Adachi-Mejia AM, et al. Exposure to movie smoking: it's relation to smoking initiation among US adolescents. Pediatrics 2005;116:1183-91.

14. Stigler MH, Perry CL, Arora M, et al. Intermediate outcomes from project MYTRI: mobilizing youth for tobacco-related initiatives in India. Cancer Epidemiol Biomarkers Prev 2007;16:1050-6.

15. Sargent JD, Worth KA, Beach M, et al. Population-based assessment of exposure to risk behaviors in motion pictures. Commun Methods Meas 2008;2:1-18.

16. Pierce JP, Choi WS, Gilpin EA, et al. Tobacco industry promotion of cigarettes and adolescent smoking. JAMA 1998;279:511-15.

17. Sargent JD, Tanski S, Stoolmiller M, et al. Using sensation seeking to target adolescents for substance use interventions. Addiction 2010:105:506-14.

18. Jackson C, Henriksen L, Foshee VA. The Authoritative Parenting Index: predicting health risk behaviors among children and adolescents. Health Educ Behav 1998;25:319-37.

19. Distefan JM, Gilpin EA, Sargent JD, et al. Do movie stars encourage adolescents to start smoking? Evidence from California. Prev Med 1999:28:1-11.

20. Tickle JJ, Sargent JD, Dalton MA, et al. Favourite movie stars, their tobacco use in contemporary movies, and its association with adolescent smoking. Tob Control 2001:10:16-22.

21. Sargent JD, Beach ML, Dalton MA, et al. Effect of seeing tobacco use in films on trying smoking among adolescents: cross sectional study. BMJ 2001;323:1-6.

22. World Health Organization. Smoke-Free Movies: From Evidence to Action. Geneva: WHO Press, 2009

23. Ministry of Health and Family Welfare. The Cigarettes and Other Tobacco Products (Prohibition of Advertisement and Regulation of Trade and Commerce Production, Supply and Distribution) Act, 2003 and Related Rules and Regulations. New Delhi: Government of India Press, 2009.

24. Biener L, Siegel M. Tobacco marketing and adolescent smoking: more support for a causal inference. Am J Public Health 2000;90:407-11.

25. Sargent JD, Dalton $\mathrm{M}$, Beach $\mathrm{M}$. Exposure to cigarette promotions and smoking uptake in adolescents: evidence of a dose-response relation. Tob Control 2000:9:163-8.

26. Chen X, Cruz TB, Schuster DV, et al. Receptivity to pro tobacco media and its impact on cigarette smoking among ethnic minority youth in California. $J$ Health Commun 2002; 7:95-111.

27. Jindal SK, Aggarwal AN, Gupta D, et al. Prevalence of tobacco use among school going youth in North Indian States. Indian J Chest Dis Allied Sci 2005:47:161-6.

28. Sargent JD, Gibson J, Heatherton TF. Comparing the effects of entertainment media and tobacco marketing on youth smoking. Tob Control 2009;18:47-53. 